\title{
Interfacial Phenomena: Reservoir Wettability in Oil Recovery
}

\author{
M. Robin ${ }^{1}$ \\ 1 Institut français du pétrole, 1 et 4, avenue de Bois-Préau, 92852 Rueil-Malmaison Cedex - France \\ e-mail: michel.robin@ifp.fr
}

Résumé - Phénomènes interfaciaux : mouillabilité des réservoirs dans la récupération pétrolière Cet article vise à illustrer l'importance de la mouillabilité dans l'industrie pétrolière et plus particulièrement dans l'exploitation des gisements.

La première partie concerne la mesure de la mouillabilité des roches réservoirs.

On montre ensuite comment des études à l'échelle microscopique permettent d'obtenir une meilleure compréhension des observations macroscopiques faites en laboratoire sur les écoulements polyphasiques en milieu poreux.

Sont présentés enfin des résultats obtenus sur des cas de réservoirs réels faisant intervenir le rôle de la minéralogie (présence et nature d'argiles) et de la distribution de la taille des pores (particulièrement dans le cas des carbonates) sur la coexistence de deux réseaux (respectivement préférentiellement mouillables à l'eau et à l'huile) dans les milieux de mouillabilité mixte.

Mots-clés : mouillabilité, milieu poreux, réservoir, carotte, pore, cryomicroscopie, ESEM.

\begin{abstract}
Interfacial Phenomena: Reservoir Wettability in Oil Recovery — This paper presents the importance of wettability in the petroleum industry, and more particularly in reservoir engineering.

It starts from the problem of reservoir wettability: how this wettability is evaluated.

It shows how microscopic scale studies help to a better understanding of macroscopic observations made in the laboratory on multiphase flows in reservoir porous material.

Finally, it presents results on actual reservoir material concerning the role of mineralogy (presence and nature of clays) and pore-size distribution (particularly in the case of carbonates) in the coexistence of two networks (water-wet and oil-wet) in so-called mixed wettability systems.

Keywords: wettability, porous medium, reservoir, core, pore, cryo-microscopy, ESEM.
\end{abstract}




\section{INTRODUCTION}

Wettability is generally considered to be one of the most important parameters influencing saturation, distribution and flow of fluids in porous media.

The minerals present in reservoir rocks are generally known as being intrinsically hydrophilic, i.e. preferentially water-wet. Until recently, many in the petroleum profession believed that all reservoirs were hydrophilic. However, problems connected with reservoir production sparked laboratory investigations that revealed the existence of reservoirs of intermediate wettability, or even clearly oil-wet.

Studies conducted by Treiber et al. (1972), Morrow (1976), Chilingar and Yen (1983) and Cuiec (1991) showed that actually, reservoirs of intermediate wettability or preferentially oil-wet were far more numerous than initially believed. They can approximately be divided into three families of comparable size: preferentially water-wet, preferentially oil-wet, and of intermediate wettability.

These studies also appear to indicate that wettability problems are more often encountered in carbonate reservoirs than in sandstone reservoirs.

As far as reservoir engineering is concerned, wettability has long been approached by global or indirect methods, such as capillary pressure curves, relative permeability curves, contact angle measurement, etc.

Studies conducted on reservoir rocks of intermediate wettability (i.e. spontaneously imbibing both water and oil) displayed heterogeneous wettability at pore scale, associated with the mineralogy or the geometry of the pore network (Fassi-Fihri et al., 1990).

The wettability of the rock is related to the affinity of its surface for water and/or oil. To get useful results in laboratory studies, it is important, not only to respect pore structure or pore geometry, by using an actual sample of the reservoir rock, but also to check that this sample presents surface properties that are representative of those prevailing in the reservoir. The quality of production forecasts determined by numerical simulation models depends to a large extent on the representativity of the measurements made in the laboratory.

The challenge consists in obtaining reservoir rock samples that are as representative as possible with regard to their surface properties. This is achieved either by preserving the original surface state or by restoration.

\section{WETTABILITY EVALUATION}

In the oil industry, wettability may be and is currently evaluated by different methods, which are mainly based on either contact angle or capillary pressure curves.

One of these methods is based on contact angle measurements. It consists of placing a drop of oil or water on the flat
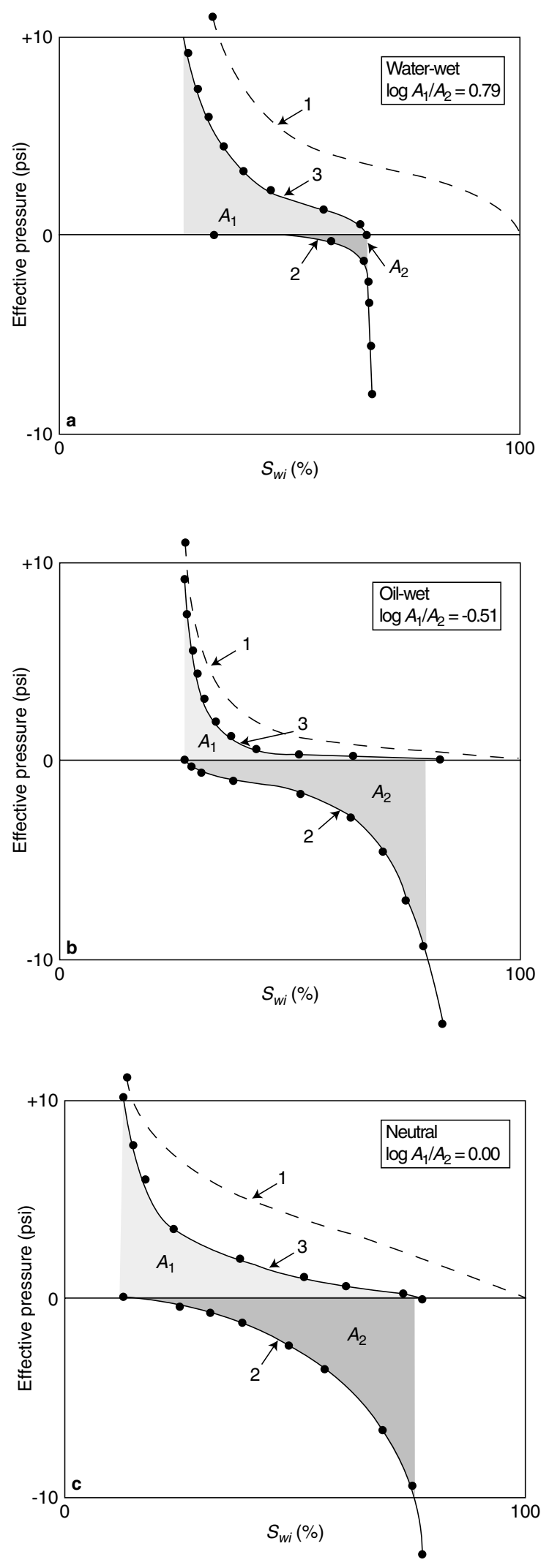

Figure 1

Effect of wettability on the capillary pressure curves: (a) water-wet, (b) oil-wet, (c) neutral (Donaldson et al., 1969). 
surface of a solid having the same mineralogical nature as the reservoir considered, and of submerging this solid in either water or oil. It is possible to operate under reservoir conditions (pressure and temperature) by the means of window cells. The problem mainly consists in selecting the minerals, getting a polished, smooth and representative surface. It is nevertheless used in some fundamental researches.

But more generally, these methods are based on capillary phenomena.

\subsection{USBM Method}

The shape of capillary pressure curves depends both on the solid wettability towards water and oil and on the pore-size distribution. It plots the capillary pressure versus the water saturation in an oil/water system (Figs. $1 a-1 c$ ). Let us define $A_{1}$ as the surface between the drainage curve and the zero pressure axis and $A_{2}$ as the surface comprised between the imbibition curve and the same axis. These surfaces are representative of the energy that is needed to inject either fluid in the porous medium: $A_{1}$ corresponds to oil and $A_{2}$ to water.

For preferentially water-wet solids, $A_{1}>A_{2}$ and $\log$ $\left(A_{1} / A_{2}\right)>0$. On the contrary, if the solid is preferentially oilwet, $A_{2}>A_{1}$ and $\log \left(A_{1} / A_{2}\right)<0$.

The logarithm of the $A_{1} / A_{2}$ ratio has been proposed to define a wettability scale. It also takes into account the influence of the pore-radius distribution.

The test performed in this way has proved to have a good reproducibility.

\subsection{Amott/IFP Method}

This method (Amott, 1959) consists of starting with a porous medium in the state of irreducible oil saturation (it means porous media is first $100 \%$ saturated with water, and then is flooded by oil until no more water is produced).

Then, the sample is immersed into oil (Fig. 2a) (volume $V_{1}=$ water spontaneously displaced by oil) and then oil is forced into the core either by centrifuging or by forced displacement (volume $V_{2}=$ water displaced by oil).

The third step consists in immersing this core into water (Fig. 2b) (volume $V_{3}=$ oil spontaneously displaced by water) and then oil is forced into the core either by centrifuging or by forced displacement (volume $V_{4}=$ oil displaced by water).

In the case of Amott method, the forced displacement is obtained by centrifuging, whereas in the case of Amott/IFP method, centrifuging is replaced by injection at a constant rate.

Amott has defined two indices: $I_{o}=V_{1} /\left(V_{1}+V_{2}\right)$ and $I_{w}=V_{3} /\left(V_{3}+V_{4}\right)$ which represent the fraction of displaceable fluid which is spontaneously displaced.

The greater the first ratio $I_{o}$, the more oil-wet the solid is, with the maximum value being one.
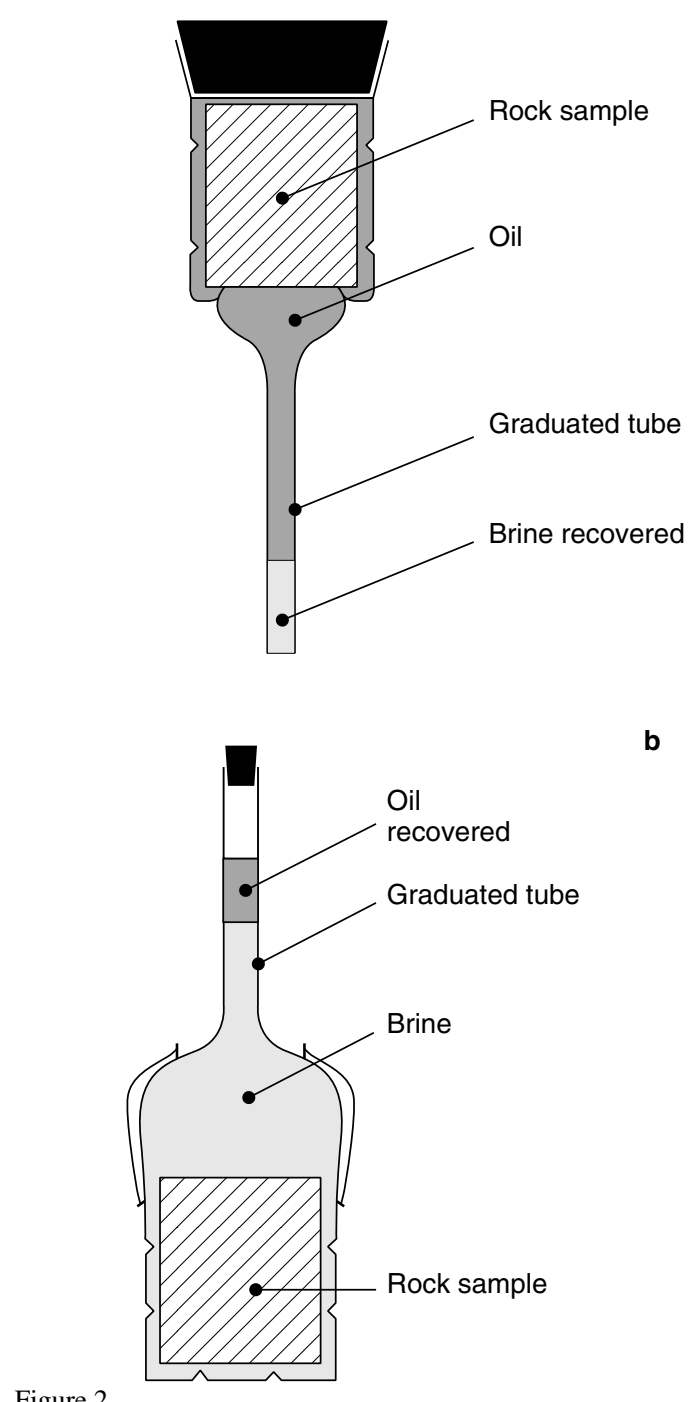

Figure 2

Apparatus for imbibition experiments in (a) oil and in (b) brine (Cuiec, 1987).

For water wettability, the role of the two ratios is reversed. When both ratios are equal to zero, the sample presents an intermediate wettability. Good reproducibility is obtained with this test.

Later, the difference of the two previous indices was proposed as a unique wettability index : $W I=I_{w}-I_{o}$.

The values of $W I$ are distributed between -1 and +1 . Negative values correspond to preferentially oil-wet systems, positive ones to water-wet systems.

Near zero, the wettability is considered as intermediate (which can be obtained either by $I_{o}=I_{w}=0$ or by $I_{w}=I_{o}=1$ which obviously does not correspond to the same wettability properties).

The USBM (US Bureau of Mines) and Amott tests have been and presently are the most widely used ones by oil and service companies. 
Many wettability configurations may occur within reservoir rock samples.

It may be homogeneous (or uniform). This means that the entire surface presents the same affinity for either water or oil. On the contrary, it may be heterogeneous, which means that some parts of the surface are preferentially water-wet while some others are preferentially oil-wet. Actually, very often reservoir rocks have the property to be able to spontaneously imbibe either water or oil (it is particularly the case for carbonate reservoir rocks).

\section{INFLUENCE OF WETTABILITY ON OIL RECOVERY}

When the wettability is uniform, it has been shown (Donaldson and Thomas, 1971) that, for a strongly hydrophilic material, the initial water wets the solid surface and saturates the small pores. If the medium is strongly oil-wet, the oil wets the solid surface, and the initial water is found in the middle of the large pores. A simplified illustration of this is given in Figures 3 and 4 (Raza et al., 1968).

Such a mechanism suggests that in a strongly oil-wet medium, oil recovery by water injection will be less effective than in a strongly water-wet medium (Anderson, 1987).

Experimentally, but also by modeling, it has been shown that oil recovery was maximum for cores of intermediate wettability, which is probably related to disconnection and trapping of the oil phase (Morrow, 1987).

The influence of wettability on multiphase flow in the porous medium and hence on oil recovery is evident. A consequence is that, to obtain reliable results, reservoir surface properties must be respected.

It is well known that there are numerous possible (and sometimes they cannot be avoided) causes of difference/ evolution in surface properties between the reservoir and the laboratory:

- the action of mud during sampling;

- temperature and pressure drop when the sample is being brought to the surface;
- contamination, oxidation, and desiccation during handling, storage, testing, etc.

Hence the necessity for oil industry to better understand the wettability phenomenon in actual reservoir material.

Some results obtained on 3D wettability studies on actual reservoir material either by cryo-SEM (cryo-Scanning Electron Microscopy) or by ESEM (Environmental Scanning Electron Microscopy) will now be presented (Robin et al., 1999).

\section{CRYO-SEM}

\subsection{Method}

Cores about $5 \mathrm{~mm}$ in diameter were cut from reservoir rocks, either sandstones or carbonates. They were cleaned by isopropanol, evacuated and dried in an oven, at $60^{\circ} \mathrm{C}$, under water vapor saturating pressure (in order to prevent drying).

The $S_{o r}$ saturation is obtained by successively evacuating the samples, $100 \%$ filling with reconstituted brine, centrifuging in oil to reach $S_{w i}$ saturation and finally centrifuging in brine. Observations were conducted either on JSM35CF or JSM6300F fitted with an EDS (Energy Dispersion Spectrometer) for elementary analysis and a cryostage and a cryo-preparation chamber Oxford CT1500. Cylindrical cores were fixed on a special sample holder, frozen in nitrogen slush, transferred to the cryo-preparation chamber where they were fractured under vacuum and coated with chromium. Each sample was then transferred to the cold stage of the microscope where it was observed at $-180^{\circ} \mathrm{C}$.

Backscattered electron images are used to study the fluid distribution. They provide a chemical contrast: each phase (oil, brine and minerals: quartz, kaolinite, etc.) appears with a different gray level depending on its mean atomic number. The lower the atomic number, the darker the phase appears. Oil appears in black, quartz and kaolinite with a light gray and brine with a mean gray level. Identification of phases is

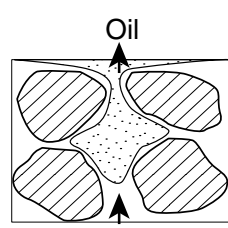

Water

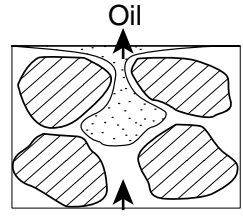

Water

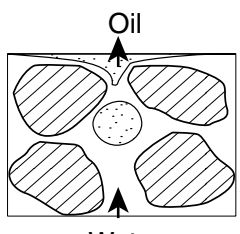

Water

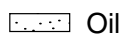

$\square \backslash$ Rock

Figure 3

Schematic diagram of imbibition process, oil displacement by water, water-wet sand (Raza et al., 1968).

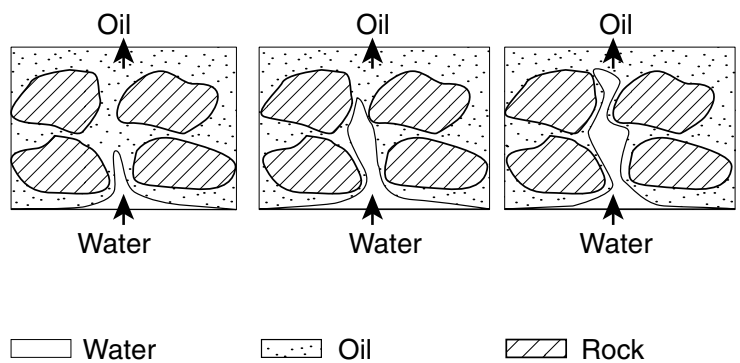

Figure 4

Schematic diagram of drainage process, oil displacement by water, oil-wet sand (Raza et al., 1968). 
confirmed by EDS elementary analyses. Sulfur is used as the tracer of oil and chlorine as the tracer of brine.

Spatial resolution is influenced both by the nature of the sample and the chosen accelerating voltage. In our laboratory, using a $15 \mathrm{kV}$ accelerating voltage, resolutions of $0.1 \mu \mathrm{m}$ in imaging and of approximately $2 \mu \mathrm{m}$ for analysis were achieved.

The procedure consists of using the secondary electron imaging mode to select a region of interest, using backscattered electrons to clearly differentiate mineral phases, oil and brine, and X-ray analysis to identify and to delimit each phase.

\subsection{Results}

It was observed (Fassi-Fihri et al., 1990) that in some clayey sandstone reservoir rocks, the kaolinite, originally preferentially water-wet, could, after aging under $S_{w i}$ conditions, constitute an oil-wet network, the quartz remaining waterwet. In carbonate rocks, it was deduced that a water-wet network was made of the smallest pores, while largest ones were at the origin of the oil-wet network.

Some examples obtained in the laboratory are presented hereafter either on sandstones (Figs. 5-9) or on carbonates (Figs. 10 and 11).

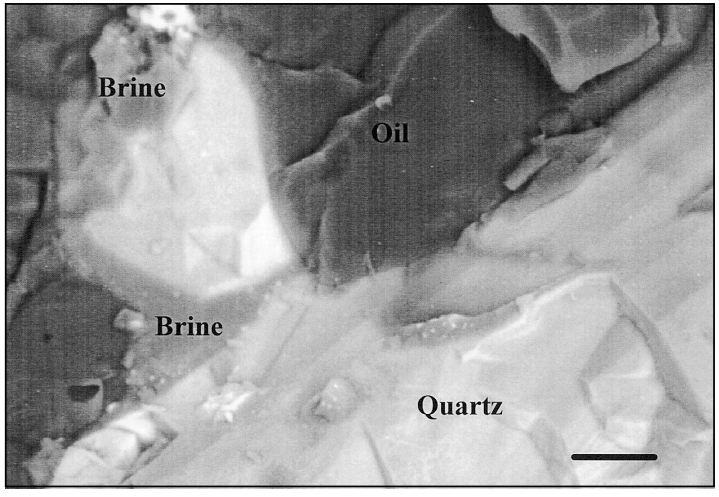

Figure 5

Fontainebleau sandstone at $S_{w i}$ saturation (water-wet). Brine appears under the form of a thick film lining the pore walls of quartz grains.

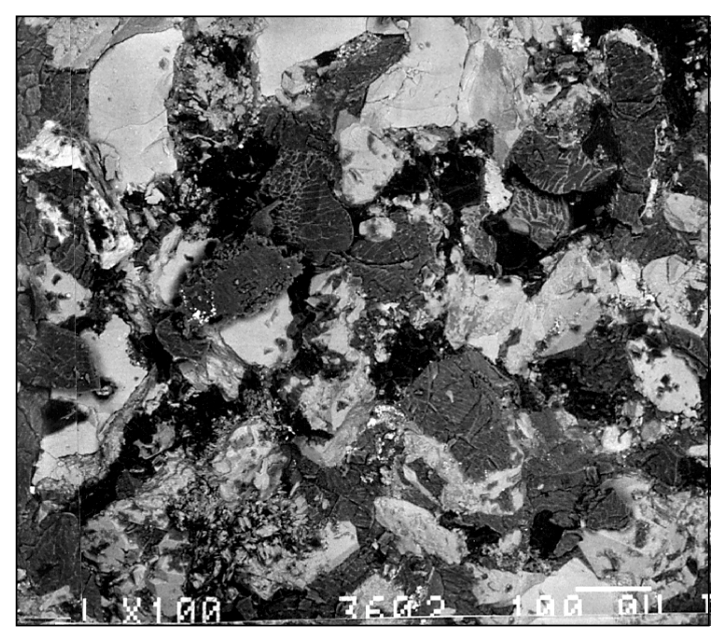

Figure 7

North Sea sandstone at $S_{o r}$ saturation. Oil appears in black.

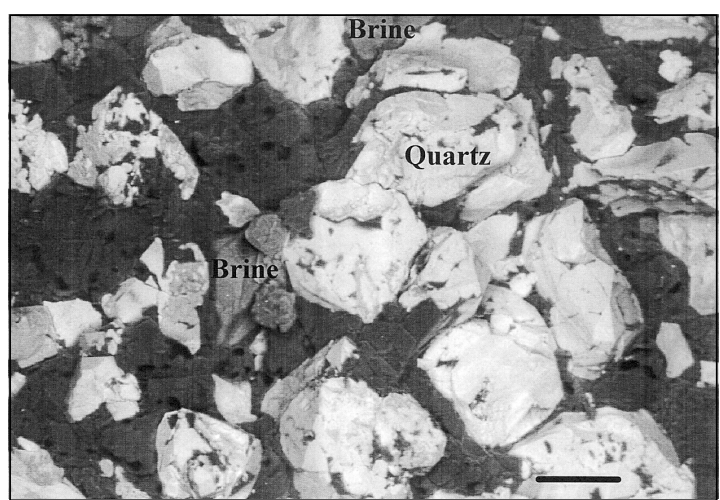

Figure 6

Silanated Fontainebleau sandstone at $S_{w i}$ saturation (oil-wet). Brine appears under the form of large globules trapped in the largest pores near constrictions.

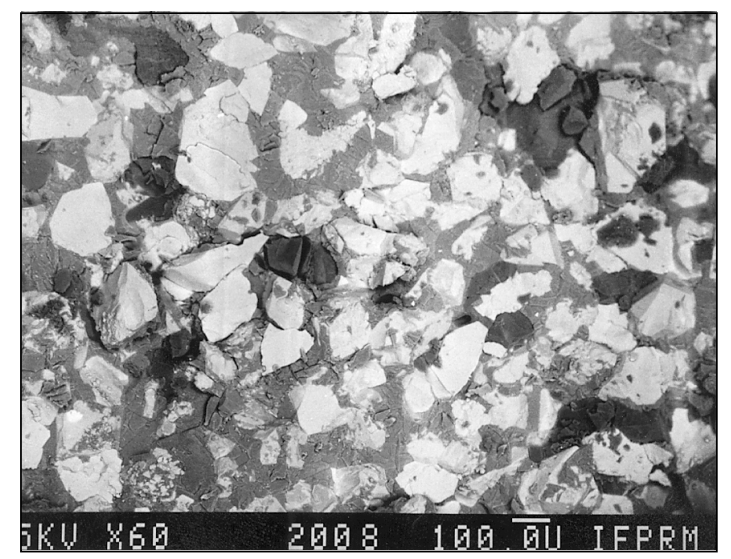

Figure 8

Clay-free sandstone at $S_{o r}$ saturation (water-wet). Oil appears as large globules in the center of the pores. 


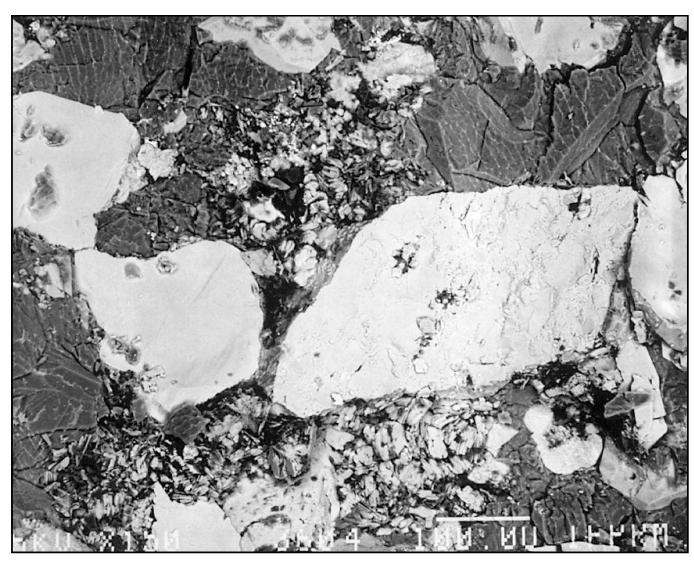

Figure 9

North Sea sandstone at $S_{\text {or }}$ saturation, non-aged (water-wet). Oil is trapped as small globules between kaolinite stacks.

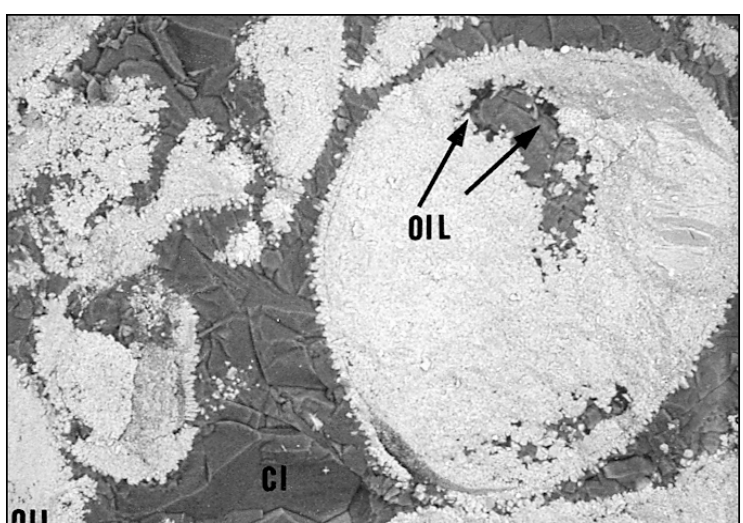

Figure 10

Middle East carbonate at $S_{o r}$ saturation. Oil lines internal walls of a peloid.

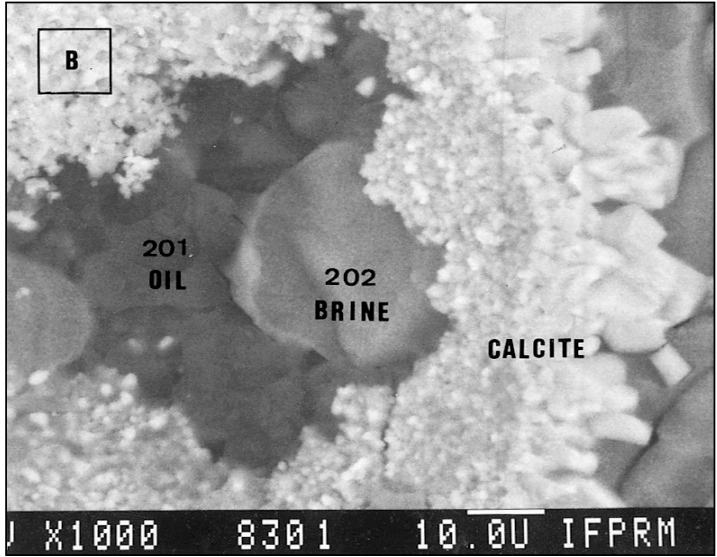

Figure 11

Middle East carbonate at $S_{o r}$ saturation. The internal part of this peloid is clearly oil-wet. Water appears in the form of globules nearby the pore walls.

\section{ESEM}

\subsection{Method}

The SEM column is equipped with a multi-stage differential pressure pumping unit. Three pumping levels allow $10^{-7}$ torr around the electron gun and a pressure of about 1 to 50 torr in the observation chamber ( 1 torr $=1 \mathrm{~mm} \mathrm{Hg}=133,32 \mathrm{~Pa}$ ).

Three apertures disposed along the column allow maintaining the pressure constant in the different stages whatever the value of the pressure and the composition of the atmosphere in the chamber. This surrounding atmosphere is generally composed of water vapor, but it can be changed according to the experiments to be made. Danilatos (1985) has developed a new electron detector operating under pressures ten thousand times higher than in a classical Everhart-Thornley detector. It involves the molecules of the environment of the chamber to amplify the signal. Ionization of the environmental gas is induced by collisions with the secondary electron emitted by the surface of the sample. Successive collisions produce a cascading amplification of the original secondary signal, which can easily be detected by a cathode.

In standard SEM, the primary electron beam creates at the surface of the non-conducting sample high negative charges. These charges cannot be evacuated, which prevents any observation, hence the technique of coating the surface of the sample by a conducting material used in standard electron microscopy. In the case of environmental microscopy, the electronic cascading multiplication results in a positive ion cloud formation, which will migrate towards the surface of the sample holder, connected to the ground. This allows evacuation of the negative charges at the surface of the sample and hence easy observation without any specific preparation or coating.

The contrast is mainly topographic. The great depth of field makes these images particularly well suited for examining surfaces. A slight chemical contrast is superimposed because of the presence of backscattered electrons. This enables a differentiation to be made between the different phases, for example, it is possible to distinguish brine (medium gray area) and oil (dark gray area). 


\subsection{Results}

\subsubsection{Operating Conditions}

The equipment used is an electroscan 3-E. A micro injection device and a Peltier-effect stage complete the microscope. So, the temperature of the sample can be adjusted between $-20^{\circ}$ and $+50^{\circ} \mathrm{C}$. Operating conditions were 6 torr and $9^{\circ} \mathrm{C}$. These conditions are just above the dew point so the water contained in the sample will not evaporate and observations can be performed in the same saturation state. On the other hand, this prevents condensation of the vapor present in the observation chamber onto the surface of the sample, which would mask the imbibition process.

The chamber is slowly evacuated to remove the excess fluid on the surface. A small amount of the imbibing fluid (a few drops) is then poured into the bottom of the vessel containing the sample.

The capillary rise of the second fluid is then observed at the upper surface of the sample.

\subsubsection{Sample Description}

\section{Sintered Glass}

It is a porous medium made of sintered glass beads (diameter about $200 \mu \mathrm{m}$ ) presenting homogeneous wettability. They are naturally water-wet. On some samples, octodecyltrichlorosilane has been grafted on the surface to make it oil-wet. These samples also present a homogeneous wettability.

\section{Mixed Wettability Sandstone-North Sea Field Reservoir (Brent Formation)}

This sandstone mainly consists of quartz, feldspar (10-15\%), mica and clays: kaolinite and illite.
Kaolinite is abundant and essentially consists of stacks of booklets of about $10 \mu \mathrm{m}$ in size. They are loosely packed within the pores.

This reservoir presents mixed wettability (it imbibes spontaneously both oil and brine: $I_{w}=0.13$ and $I_{o}=0.70$; $\left.W I=I_{w}-I_{o}=-0.57\right)$.

\section{Mixed Wettability Carbonate—Middle East Reservoir}

Samples consist of a packstone, with peloids, foraminifera, a few ooids and aggregates. Grains are rough, their size is rather heterogeneous (about 100-125 $\mu \mathrm{m}$ for average grain diameter). Porosity consists of:

- the mesoporosity, which is either intergranular (dominant) or intragranular (essentially included in foraminifera cavities);

- the microporosity, which is essentially associated with cement crystals.

Wettability was estimated to be mixed, with spontaneous displacement in both oil and brine $\left(I_{w}=0.11\right.$ and $I_{o}=0.92$; $\left.W I=I_{w}-I_{o}=-0.81\right)$.

\subsubsection{Oil Imbibition in Brine Saturated Samples}

The oil phase consisted in normal dodecane $\left(\mathrm{C}_{12} \mathrm{H}_{26}\right)$.

\section{Silanated Sintered Glass Cores}

These cores are strongly oil-wet. In a water/oil system, the contact angle measured in oil phase is about $0^{\circ}$. As soon as oil has been poured into the bottom of the vessel containing the sample, a dark ring appears at the edge of the beads (Fig. 12). Its size increases very quickly and it spreads on the surface of the grain. A few seconds later, the entire surface of the sample is covered.

This first step allowed checking the feasibility of the experiments and defined the most favorable operating conditions to work on real systems.

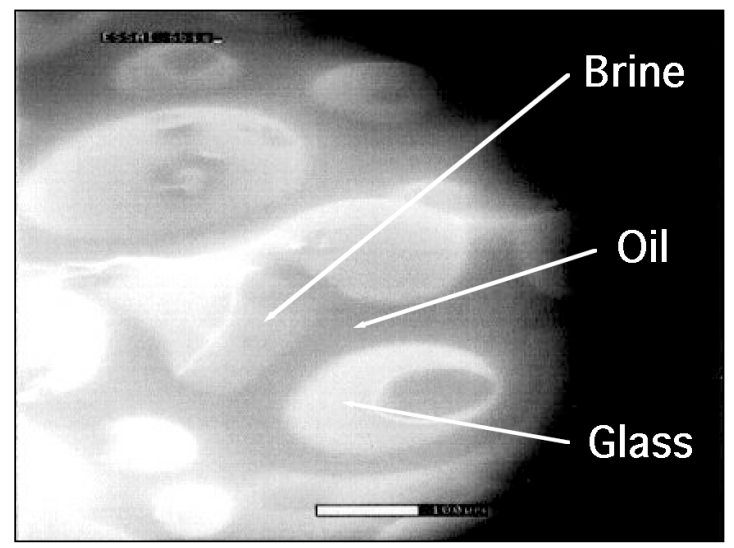

Figure 12

Oil imbibition in oil-wet sintered glass.

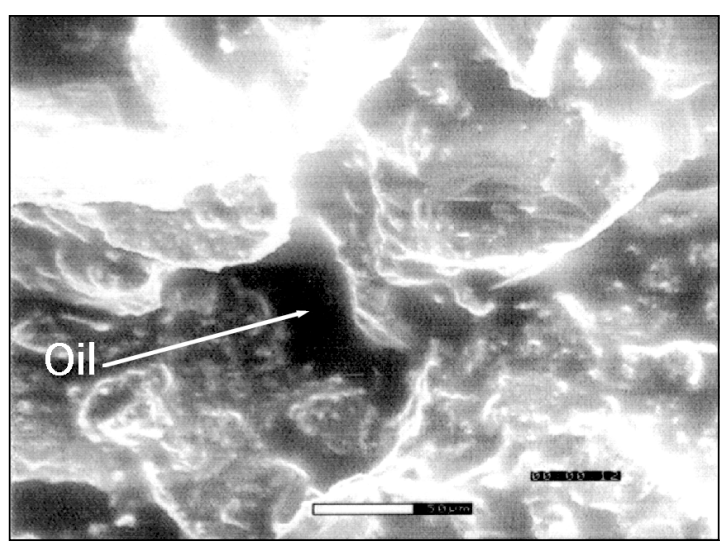

Figure 13

North Sea sandstone. Appearance of oil in a pore filled by kaolinite. 


\section{Mixed Wettability Sandstone}

In the case of this sandstone (Fig. 13), oil has been observed to appear at the upper surface through kaolinite areas (either loosely packed within the pore or covering quartz grains).

\section{Mixed Wettability Carbonate}

Oil first appears in the largest pores $(>10 \mu \mathrm{m})$ where capillary rise occurs very quickly (Fig. 14). On intermediate size pores, the phenomenon was more gradual and was delayed, compared to what happens in the largest pores.

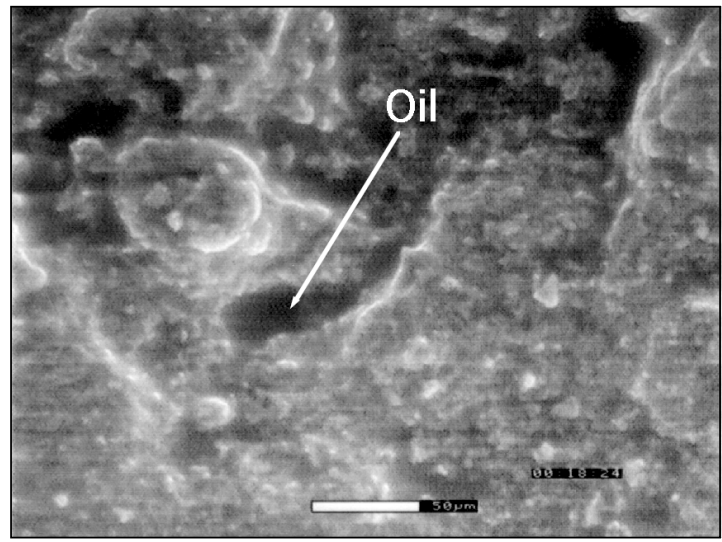

Figure 14

Middle East carbonate. Appearance of oil in a large pore.

\section{CONCLUSIONS}

There is an important influence of wettability on multiphase flow in porous medium and hence on oil recovery.

Consequently, there is a necessity for oil industry to better understand the wettability phenomenon in actual reservoir material.

To obtain reliable results, it is very important to respect reservoir surface properties.

Both cryo-SEM and ESEM techniques must be considered as very efficient tools for studying complex and heterogeneous systems. They offer new perspectives for the understanding of the influence of surface properties distribution on two- and three-phase displacements.

Both techniques enhance our understanding of the different interactions within the porous media. These interactions depend on wettability, which governs the fluid distribution.
ESEM allows visualization of fluid capillary rise in rock samples. It allows the observation of dynamic experiments, for instance a wetting fluid imbibition in presence of a nonwetting fluid.

Experiments confirmed the static observations obtained by cryo-SEM: wettability heterogeneity at the pore scale, and the influence of composition, size and distribution of minerals on physico-chemical behavior of the sample towards fluids. In the mixed wettability sandstone, oil capillary rise seems to occur through kaolinite zones. In case of carbonate, oil capillary rise seems to occur through the largest pores.

\section{REFERENCES}

Amott, E. (1959) Pet. Trans. AIME, 216, 156-162.

Anderson, W.G. (1987) Wettability Literature Survey. J. Pet. Technol., December, 1605-1622.

Chilingar, G.V. and Yen, T.F. (1983) Some Notes on Wettability and Relative Permeabilities of Carbonate Reservoir Rocks. Energy Sources, 7, 1, 67-75.

Cuiec, L. (1987) in Proc. of the North Sea Oil and Gas Reservoirs Seminar, Trondheim, Norway, December 2-4, 1985, Graham and Trotman (eds.), London, 193-207.

Cuiec, L. (1991) in Interfacial Phenomena in Petroleum Recovery, Morrow, N. (ed.), Marcel Dekker, New York.

Danilatos, G. (1985) Micron and Microscopia Acta, 14, 307-319.

Donaldson, E.C. and Thomas, R.D. (1971) 46th Annual Fall Meeting, New Orleans, LA, October 3-6, Paper SPE 3555.

Donaldson, E.C., Thomas, R.D. and Lorenz, P.B. (1969) SPE J., March, 13-20.

Morrow, N.R. (1976) Capillary Pressure Correlations for Uniformly Wetted Porous Media. J. Can. Pet. Technol., 49-69.

Morrow, N.R. (1987) in Proc. of the North Sea Oil and Gas Reservoirs Seminar, Trondheim, Norway, December 2-4, 1985, Graham and Trotman (eds.), London, 179-191.

Fassi-Fihri, O., Robin, M. and Rosenberg, E. (1990) Visualization of Oil and Brine in Porous Media by Cryo-Scanning Electron Microscopy. Fundamentals of Fluid Transport in Porous Media, Proc. of Institut Français du Pétrole Exploration and Production Research Conference, Arles, France, May 14-18, Vol. 1, 15-18.

Raza, S.H., Treiber, L.E. and Archer, D.L. (1968) Producers Monthly, 32, 4, 2-7.

Robin, M., Combes, R. and Rosenberg, E. (1999) Cryo-SEM and ESEM: New Techniques to Investigate Phase Interactions within Reservoir Rocks. 1999 SPE Annual Technical Conference and Exhibition, Houston, TX, Paper SPE 56829.

Treiber, L.E., Archer, D.L. and Owens, W.W. (1972) Laboratory Evaluation of the Wettability of 50 Oil Producing Reservoirs. SPE J., 12, 531-540.

Final manuscript received in November 2000 